\title{
Peer review in the CiSE RR Track
}

\author{
Lorena A. Barba ${ }^{1}$ and George K. Thiruvathukal ${ }^{2}$ \\ ${ }^{1}$ George Washington University \\ ${ }^{2}$ Loyola University Chicago
}

May 5, 2020

In our editorial launching the new Reproducible Research Track in CiSE (Barba and Thiruvathukal, 2017), we promised to explore innovations to the peer-review process. Because we require articles submitted to this track to adhere to practices that safeguard reproducibility, we must review for these aspects deliberately. For each submission, a reproducibility reviewer will be charged with checking availability, quality and usability of digital artifacts (data, code, figures). This reviewer (sometimes one of the track editors) will be known to the authors, and may interact with the authors during the review-for example, opening issues on a code repository. For this service, we ask that the authors recognize the reviewer in the article's acknowledgements section.

The double-open review model seems natural when treating code and data. This is perhaps an effect of the social norms in open-source-software communities. Open peer review of the manuscript itself still raises concerns, however. While there's some evidence that open reviews are of higher quality and more courteous (Walsh et al., 2000), early career researchers may shy away from the idea of having to write a critical review of a senior researcher's work, fearing career retribution. A recent study of attitudes and experiences among researchers (Ross-Hellauer et al., 2017) found that while $60 \%$ of respondents believed open reports would increase review quality, they were less keen on open identities, and $74 \%$ believed reviewers should be allowed to opt in to making their identity open.

In the CiSE RR track, we want to promote transparency, and opening up peer review has to be a part of that. When requesting reviews, we will tell the potential reviewers that they will have the ability to opt-in to have their identity known to the author. Upon sending a decision letter that requests a revision, we will ask the authors to make a summary of the reviewers' contribution to improving their paper, and include that in an extended acknowledgements section. We will also encourage the reviewers to deposit their review report in an archival repository that assigns a digital object identifier, DOI (such as Figshare or Zenodo). For point-by-point responses by the authors, we'll also suggest a deposit with a DOI. Both reports can be cited in the final article, and elsewhere - we see this as a superior solution to supplementary materials (which are hard to find and not independently citable).

As editors for the RR track, we will use our judgement and authority to moderate the process, making the extended acknowledgement a condition of acceptance. When reviewers opt in to open identity, we'll provide guidance on using repositories to make an open report. We already require manuscripts submitted to the RR track to deposit a pre-print, which coupled with open reports will transparently preserve the full history of a published article.

\section{References}

Lorena A. Barba and George K. Thiruvathukal. Reproducible Research for Computing in Science \& Engineering. Computing in Science \& Engineering, 19(6):85-87, nov 2017. doi: 10.1109/mcse.2017.3971172. 
URL https://doi.org/10.1109\%2Fmcse.2017.3971172.

Tony Ross-Hellauer, Arvid Deppe, and Birgit Schmidt. Survey on open peer review: Attitudes and experience amongst editors authors and reviewers. PLOS ONE, 12(12):e0189311, dec 2017. doi: 10.1371/journal. pone.0189311. URL https://doi.org/10.1371\%2Fjournal. pone. 0189311.

Elizabeth Walsh, Maeve Rooney, Louis Appleby, and Greg Wilkinson. Open peer review: A randomised controlled trial. British Journal of Psychiatry, 176(01):47-51, jan 2000. doi: 10.1192/bjp.176.1.47. URL https://doi.org/10.1192\%2Fbjp.176.1.47. 